\title{
JET-AMBIENT INTERACTION OF A SUPERSONIC, RADIATIVELY-COOLED JET IN LABORATORY EXPERIMENTS
}

\author{
F. Suzuki-Vidal ${ }^{1}$, M. Bocchi ${ }^{1}$, S.V. Lebedev ${ }^{1}$, J. Skidmore ${ }^{1}$, \\ G. Swadling ${ }^{1}$, G. Burdiak ${ }^{1}$, A.J. Harvey-Thompson ${ }^{1}$, P. de Grouchy ${ }^{1}$, \\ S.N. Bland ${ }^{1}$, G.N. Hall ${ }^{1}$, E. Khoory ${ }^{1}$, L. Pickworth ${ }^{1}$, L. Suttle ${ }^{1}$, \\ J.P. Chittenden ${ }^{1}$, M. Krishnan ${ }^{2}$, K. Wilson-Elliot ${ }^{2}$, R. Madden ${ }^{2}$, \\ A. Ciardi ${ }^{3}$ and A. Frank ${ }^{4}$
}

\begin{abstract}
The formation of supersonic, radiatively cooled plasma jets with applications to laboratory astrophysics has been an active area of research on the MAGPIE generator. One of the ways of producing astrophysically-relevant jets in the laboratory is by using the ablation of plasma from a radial foil Z-pinch. In this configuration a 1.4 MA, $250 \mathrm{~ns}$ current pulse is introduced into an aluminium disk with a thickness of $15 \mu \mathrm{m}$. The ablated plasma from the foil converges on the axis, producing a steady and collimated jet with a typical axial velocity of $\sim 100 \mathrm{~km} / \mathrm{s}$. The setup allows for the addition of argon above the foil for jet-ambient interaction studies. The interaction is characterised by the formation of several shock features, which are presented and discussed from experimental data and numerical simulations.
\end{abstract}

\section{Introduction}

High-energy density laboratory plasma astrophysics deals with the experimental modelling of astrophysical processes (for a review see e.g. Remington et al. 2006), and particularly the physics of highly collimated, highly supersonic jets from young stars (Hartigan et al. 2011, see T. Ray, this volume). Their study is challenging, as

\footnotetext{
${ }^{1}$ Imperial College London, Blackett Laboratory, Prince Consort Road, London SW7 2BW, UK

2 Alameda Applied Sciences Corporation, San Leandro, CA 94577, USA

${ }^{3}$ LERMA, Université Pierre et Marie Curie, Observatoire de Paris et École Normale Supérieure, UMR 8112 CNRS, France

${ }^{4}$ Department of Physics and Astronomy, University of Rochester, Rochester, NY 14627-0171, USA
} 
(a)

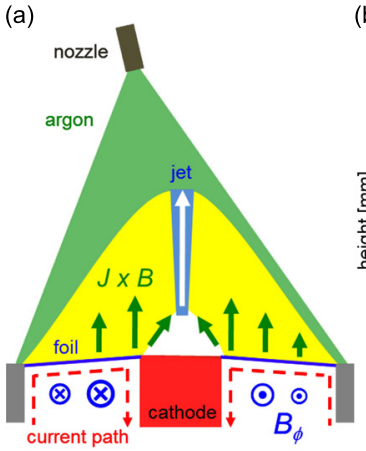

(b)

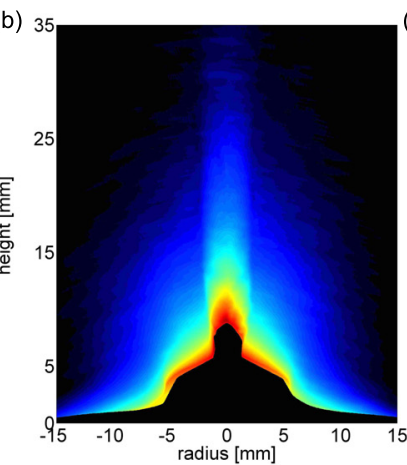

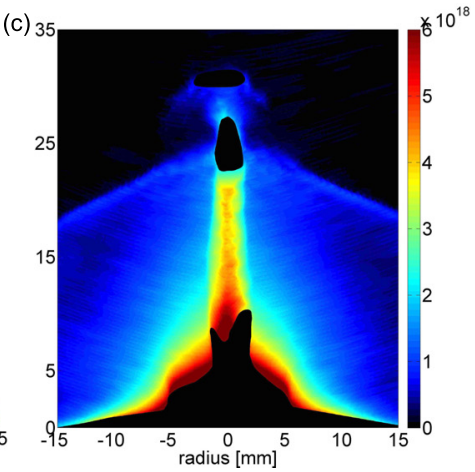

Fig. 1. (a) Schematic setup for jet-ambient interaction using a radial foil. (b)-(c) Lineelectron density results (in units of $\mathrm{cm}^{-2}$, at $430 \mathrm{~ns}$ ) showing the jet in vacuum and in argon respectively. The height is measured from the initial position of the cathode.

experiments need to include the effects of radiative cooling, magnetic fields, and the formation of complex shock features. Scaled experiments using high-power lasers and high-current generators, however, have managed to reproduce particular aspects of these jets, e.g. their launching mechanisms (Lebedev et al. 2005, see S. Lebedev, this volume), and the propagation of the jet with the interstellar medium (Nicolaï et al. 2010). The work presented here aims to extend the latter, by studying the interaction of a supersonic aluminium jet propagating into a cold, neutral argon gas.

\section{Experimental and numerical setup}

A plasma jet is produced using a radial foil Z-pinch (Suzuki-Vidal et al. 2010, shown schematically in Fig. 1a), a $\mu$ m thick aluminium disk held between two concentric cylindrical electrodes and subjected to the $\sim 1.4 \mathrm{MA}, 250 \mathrm{~ns}$ current from the MAGPIE pulsed-power generator (Mitchell et al. 1996). The current flows along the central electrode (diameter of $6.35 \mathrm{~mm}$ ), and radially along the foil. The current Ohmically heats the foil and, within the first $~ 100 \mathrm{~ns}$ converts it into plasma. The current path produces a $\mathrm{J} \times \mathrm{B}$ force which ablates plasma in a direction perpendicular to the surface of the foil. A jet is produced as plasma convergences on the axis of the foil, due to radial pressure gradients due to the lack of ablation in this region. The interaction of the jet with an ambient medium was achieved by injecting cold, neutral argon gas above the initial position of the foil using a solenoid-valve with a supersonic gas nozzle (Krishnan et al. 2009). The tip of the nozzle was positioned $55 \mathrm{~mm}$ above the foil and $15 \mathrm{~mm}$ from the axis in order to prevent direct impact of the jet onto the nozzle. The chosen position of the valve insures argon covers the entire foil surface during the current discharge. The valve was opened typically $\sim 350 \mu$ s before the start of the current pulse, 
with a duration of $\sim 200 \mu \mathrm{s}$. The nozzle had a length of $8 \mathrm{~mm}$, a throat diameter of $\sim 300 \mu \mathrm{m}$, and an exit diameter of $\sim 2 \mathrm{~mm}$, releasing argon with a Mach number of $\mathrm{M} \sim 9$. The measured gas density at the position of the foil was estimated to be $\rho \sim 3-5 \times 10^{-3} \mathrm{~kg} / \mathrm{m}^{3}$, equivalent to a number density of $\mathrm{N} \sim 4-7 \times 10^{16} \mathrm{~cm}^{-3}$.

Numerical simulations of the experiments were performed using the GORGON code (Ciardi et al. 2007, see also Ciardi, this volume), an explicit, parallel code designed to solve the resistive MHD equations on a three-dimensional Cartesian grid. The resolution of the computational grid was $200 \mu \mathrm{m}$. The foil was initialised as an aluminium disk with a thickness of 2 computational cells, and the transition from solid state into plasma started only after the foil reached the melting temperature.

The dimensionless parameters that describe the jet in the experiments are comparable to those found in young stellar jets. In experiments the jet have an internal Mach number of $\mathrm{M} \sim 3$, with a jet-to-ambient density ratio $\eta>1$. In young stellar jets these numbers are typically $\mathrm{M}>10$ and $\eta \sim 10$ respectively (Remington et al. 2006). It should be noted that in both cases there is a strong effect of radiative cooling in the jet, i.e. the cooling parameter $\chi<1$.

\section{Results from jet-ambient interaction}

The main features of a jet from a radial foil in vacuum and with the addition of argon are shown in Figures 1b-c respectively. Both images were obtained at $\sim 430$ ns using optical laser interferometry $(\lambda=532 \mathrm{~nm}, \sim 0.3 \mathrm{~ns}$ pulse duration) and represent 2-D maps of line-electron density, i.e. electron density integrated along the length of the plasma region. Without the presence of argon (Fig. 1b), the jet is a well defined, highly-collimated column with a typical diameter of $\sim 4 \mathrm{~mm}$, and an aspect ratio (jet length to radius) $>20$. Abel inversion of these data results in typical electron densities in the jet of $\sim 10^{19} \mathrm{~cm}^{-3}$, surrounded by lower density plasma with a typical electron density of $<10^{18} \mathrm{~cm}^{-3}$. Results from optical Thomson scattering show the jet is supersonic $(\mathrm{M} \sim 3)$ and radiatively cooled, i.e. its cooling parameter $\chi<1$.

The main features of the jet interacting with an argon ambient are presented in Figure 1c. A jet with similar characteristics to the case in vacuum is observed, however the presence of argon leads to the formation of two prominent shock features. The first shock feature extends from the tip of the jet towards the edges of the foil in an "inverted cone" shape. The second shock feature is observed at this time ahead of the tip of the jet, forming a quasi-hemispherical shape and more resemblant to a bow-shock. The dynamics of the formation of these two shock features can be observed in detail in Figure 2a from time-resolved images recording the self-emission of the plasma in the XUV $(\mathrm{h} \nu>30 \mathrm{eV})$. These results allow measuring the axial tip velocities of both shock features, resulting in approximately constant axial velocities of $\mathrm{V}_{\text {jet }} \sim 70 \mathrm{~km} / \mathrm{s}$ and $\mathrm{V}_{\text {bow }} \sim 90 \mathrm{~km} / \mathrm{s}$, i.e. the bowshock moves faster by $\sim 30 \%$.

Numerical simulations of the XUV emission are presented in Figure 2b, and show a very good agreement with the experimental data. The simulations indicate 
(a) $175 \mathrm{~ns}$

$$
230 \mathrm{~ns}
$$

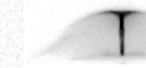

(b)
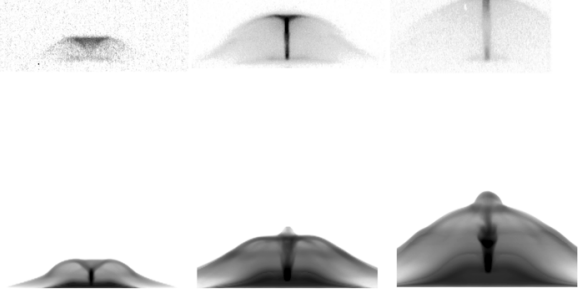

$290 \mathrm{~ns}$
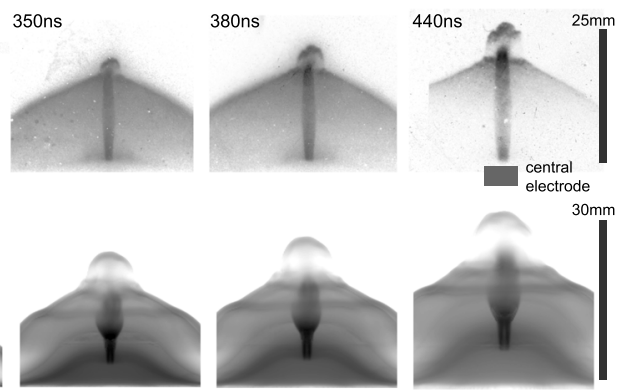

Fig. 2. Time-resolved, XUV self-emission images of the jet interacting with argon from (a) experiments, (b) numerical simulations.

that the plasma flow from the jet and from the foil converge at the tip of the jet due to the presence of a radial component of the $\mathrm{J} \times \mathrm{B}$ force, i.e. some fraction of the current flows along the jet and along the inverted-conical shock. The convergence of current at the tip of the jet produces a compressing effect, which increases the density, temperature and pressure in this region, and explains the strong emission observed at the tip of the jet on the XUV images. The formation of the bowshock ahead of the tip of the jet is due to the localised increase of pressure in this region, which creates strong axial pressure gradients, causing an acceleration of the plasma above it and producing a "nozzle-like" configuration. This allows plasma to propagate ahead of the tip of the jet at a higher velocity and interact with undisturbed argon.

\section{Future work}

The experiments presented here open new possibilities for the study of supersonic, radiatively-cooled plasma jets relevant to the physics of jets from young stars, and particularly to the physics of the interaction of such jets with the interstellar medium. The formation and interaction of the bow-shock ahead of the jet with the undisturbed argon ambient is very promising for the investigation of the effects of radiative cooling on the properties of forming working surfaces, which is highly relevant to astrophysical jets.

This work was supported by the EPSRC Grant no. EP/G001324/1, by the NNSA under DOE Cooperative Agreements No. DE-F03-02NA00057 and No. DE-SC-0001063, by DOE SBIR Grant DE-FG02-08ER85030, and by a Marie Curie European Reintegration grant.

\section{References}

Remington, B.A., Drake, R.P., \& Ryutov, D.D., 2006, Rev. Mod. Phys., 78, 755

Hartigan, P., Frank, A., Foster, J.M., et al., 2011, ApJ, 736, 29 
Lebedev, S.V., Ciardi, A., Ampleford, D.J., et al., 2005, MNRAS, 361, 97

Nicolaï, P., Stenz, C., Tikhonchuk, V., et al., 2010, Phys. Plasmas, 17, 112903

Suzuki-Vidal, F., Lebedev, S.V., et al., 2010, Phys. Plasmas, 17, 112708

Mitchell, I.H., Bayley, J.M., Chittenden, J.P., et al., 1996, Rev. Sci. Instrum., 67, 1533

Krishnan, M., Bures, B., Madden, R., Blobner, F., \& Elliott, K.W., 2009, Am. Inst. Phys. Conf. Ser., 1194, 56

Ciardi, A., Lebedev, S.V., Frank, A., et al., 2007, Phys. Plasmas, 14, 056501 